\title{
Critical Behavior near the Ferromagnetic to Paramagnetic Phase Transition in $\mathrm{Y}_{8} \mathrm{Co}_{62} \mathrm{~B}_{30}$ Amorphous Alloy
}

\author{
Z. ŚNIADECKI ${ }^{a, *}$, J. MARCIN $^{b}$ AND B. IDZIKOWSKI ${ }^{a}$ \\ ${ }^{a}$ Institute of Molecular Physics, Polish Academy of Sciences, M. Smoluchowskiego 17, 60-179 Poznań, Poland \\ ${ }^{b}$ Institute of Experimental Physics, Slovak Academy of Sciences, Watsonova 47, 04001 Košice, Slovakia \\ The $\mathrm{Y}_{8} \mathrm{Co}_{62} \mathrm{~B}_{30}$ amorphous alloy is a collinear ferromagnet. The Arrott plot, the Kouvel-Fisher method, \\ critical isotherm analysis and magnetic field dependence of maximum magnetic entropy changes were the main \\ analytical tools used to determine the nature of the ferromagnetic/paramagnetic phase transition. Values of critical \\ exponents $(\beta, \gamma$, and $\delta)$ were calculated and were convergent with those characteristic for mean-field model. It \\ suggests that long range ferromagnetic interactions are playing main role in investigated alloy and the phase \\ transition was confirmed to be of a second order according to the Banerjee criterion.
}

DOI: 10.12693/APhysPolA.131.967

PACS/topics: $75.40 . \mathrm{Cx}, 75.50 . \mathrm{Kj}, 75.30 . \mathrm{Sg}$

\section{Introduction}

It has been shown that addition of metalloid is crucial for improvement of glass forming ability and thermal stability of amorphous phase for transition metal based systems [1]. Boron plays significant role as it obstructs the crystallization process and $\mathrm{Co}-\mathrm{B}$ or $\mathrm{RE}-\mathrm{Co}-$ $\mathrm{B}(\mathrm{RE}=$ rare earth element $)$ amorphous alloys can be obtained in wide composition range [2,3]. Further development of the rare earth-transition metal multicomponent amorphous alloys is important for understanding of their magnetic behavior and from the point of view of possible applications, for instance magnetic refrigeration [4]. The magnetic properties are mainly predefined by the existence of localized $4 f$ moments of lanthanide atoms and $3 d$ moments of transition metal. The $\mathrm{Y}-\mathrm{Co}_{-}$ $\mathrm{B}$ alloys are typical collinear ferromagnets as shown before [2]. Investigations on the $\mathrm{RE}_{8} \mathrm{Co}_{62} \mathrm{~B}_{30}$ amorphous alloys $(\mathrm{RE}=\mathrm{Tb}, \mathrm{Ho}$ or $\mathrm{Y})$ have shown the influence of heavy rare earth substitution on the changes of magnetic and magnetocaloric properties [2], but determination of the character of phase transformation at around the Curie temperature $T_{\mathrm{C}}$ is another important aspect [5]. Type of the transition is believed to be of the second order [2]. In this study we investigate the critical phenomena in $\mathrm{Y}_{8} \mathrm{Co}_{62} \mathrm{~B}_{30}$ (the representative with non-magnetic rare earth component) basing on different approaches including the Arrott plot, the Kouvel-Fisher method and the critical isotherm analysis. Calculations provide information on the Curie temperature, the $\beta, \gamma$, and $\delta$ critical exponents, which finally can enrich our knowledge on the nature and physics underlying this phase transformation.

\section{Experimental}

Master alloy of $\mathrm{Y}_{8} \mathrm{Co}_{62} \mathrm{~B}_{30}$ was synthesized by arcmelting of high purity elements (3N or more) in argon

*corresponding author; e-mail: sniadecki@ifmpan.poznan.pl atmosphere. Ingot was melt-spun on a copper wheel rotated with the surface velocity of $40 \mathrm{~m} / \mathrm{s}$ and obtained ribbons were $20-30 \mu \mathrm{m}$ thick. The structure of the ribbons was examined with the use of X-ray diffractometer with Co $K_{\alpha}$ radiation in the Bragg-Brentano geometry. The magnetic field dependence of magnetization was measured by SQUID magnetometer.

\section{Results and discussion}

Fully amorphous structure has been confirmed in our previous paper basing on X-ray diffraction measurements [2]. Scaling hypothesis was used for calculations utilizing $M(H)$ curves as the input data. The critical behavior of a second-order transition can be characterized by a set of critical exponents $[6,7]$. Exponent $\beta$ is connected with the spontaneous magnetization $M_{s p}, \gamma$ with the inverse initial magnetic susceptibility $\chi_{0}^{-1}$ and finally $\delta$ with the critical magnetization isotherm at the Curie temperature. They are defined as follows $[8,9]$ :

$$
\begin{aligned}
& M_{s p}(T)=M_{0}(-\varepsilon) \beta, \text { for } \varepsilon<0, \\
& \chi_{0}^{-1}(T)=\left(h_{0} / M_{0}\right) \varepsilon^{\gamma}, \text { for } \varepsilon>0, \\
& M=D H^{1 / \delta}, \text { for } \varepsilon=0,
\end{aligned}
$$

where $\varepsilon$ equal to $\left(T-T_{\mathrm{C}}\right) / T_{\mathrm{C}}$ is the reduced temperature, $M_{0}, h_{0}$ and $D$ are critical amplitudes, $M$ is magnetization and $H$ is applied magnetic field. Additionally, Widom scaling relation [10] links all critical exponents:

$$
\delta=1+\gamma / \beta \text {. }
$$

Figure 1 shows the isothermal magnetization curves of $\mathrm{Y}_{8} \mathrm{Co}_{62} \mathrm{~B}_{30}$ measured in the temperature range from 170 to $260 \mathrm{~K}$, presented in the form of the Arrott plot. Red and blue curves represent the ferromagnetic and paramagnetic regions, respectively, with black curve corresponding to critical region. Each isotherm is close to be linear in moderate and high magnetic fields and therefore mean field (MF) model was assumed to be applicable for further analysis and modifications of the Arrott plot were not considered. The phase transition was confirmed to be 
of a second order according to the Banerjee criterion [11], where the positive sign of the $M^{2}(H / M)$ slope was found for each isotherm.

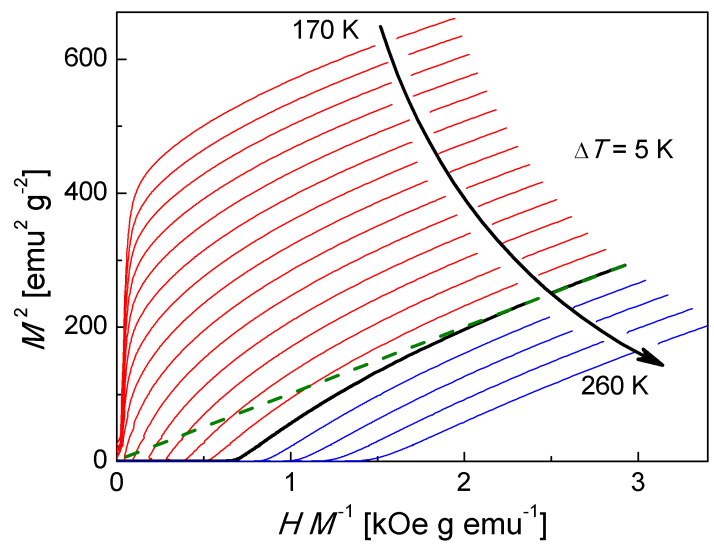

Fig. 1. Arrott plot for $\mathrm{Y}_{8} \mathrm{Co}_{62} \mathrm{~B}_{30}$ amorphous alloy from magnetization isotherms measured with interval of $5 \mathrm{~K}$. Dashed line is the guide for eye only and represents the extrapolation of critical isotherm in high magnetic field.

The spontaneous magnetization $M_{s p}$ and the inverse initial susceptibility $\chi_{0}^{-1}$ were determined from the intersections of the linear extrapolation of high field regions of isotherms with the $M^{2}$ and $H / M$ axes, respectively. Obtained temperature dependences of $M_{s p}$ and $\chi_{0}^{-1}$ are presented in Fig. 2. Experimental points (open circles) were fitted with polynomial (red line) and linear (blue line) functions for spontaneous magnetization and inverse initial magnetic susceptibility, respectively. Value of $T_{\mathrm{C}}$ was estimated from intersection of extrapolated curves with temperature axis. Values determined from paramagnetic and ferromagnetic regions are similar to each other and equal to $239.0 \pm 0.5 \mathrm{~K}$ and $242.8 \pm 0.5 \mathrm{~K}$, respectively.

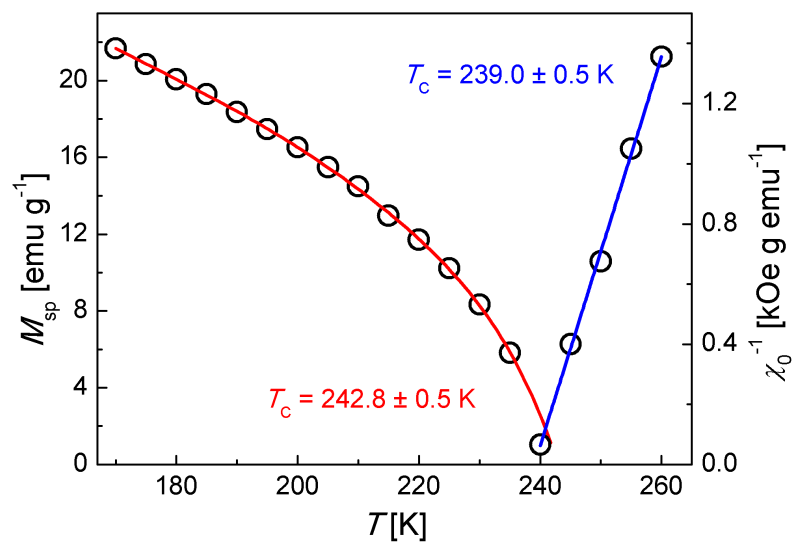

Fig. 2. Temperature dependence of the spontaneous magnetization $M_{s p}$ (left axis) and the inverse initial magnetic susceptibility $\chi_{0}^{-1}$ (right axis) for $\mathrm{Y}_{8} \mathrm{Co}_{62} \mathrm{~B}_{30}$ amorphous alloy.

The Kouvel-Fisher method [9] was used to determine critical exponents with high accuracy. It expresses dependences presented in Eqs. (1) and (2) in the following form:

$$
\begin{aligned}
& M_{s p}(T) /\left(\mathrm{d} M_{s p}(T) / \mathrm{d} T\right)=\left(T-T_{\mathrm{C}}\right) / \beta, \\
& \chi_{0}^{-1}(T) /\left(\mathrm{d} \chi_{0}^{-1}(T) / \mathrm{d} T\right)=\left(T-T_{\mathrm{C}}\right) / \gamma .
\end{aligned}
$$

According to Eqs. (5) and (6), values of $1 / \beta$ and $1 / \gamma$ can be determined from the slopes of the linear fits to the temperature dependences of $M_{s p}(T) /\left(\mathrm{d} M_{s p}(T) / \mathrm{d} T\right)$ and $\chi_{0}^{-1}(T) /\left(\mathrm{d} \chi_{0}^{-1}(T) / \mathrm{d} T\right)$, respectively, as shown in Fig. 3. The Curie temperatures were obtained from intersections of fitted curves with the temperature axis.

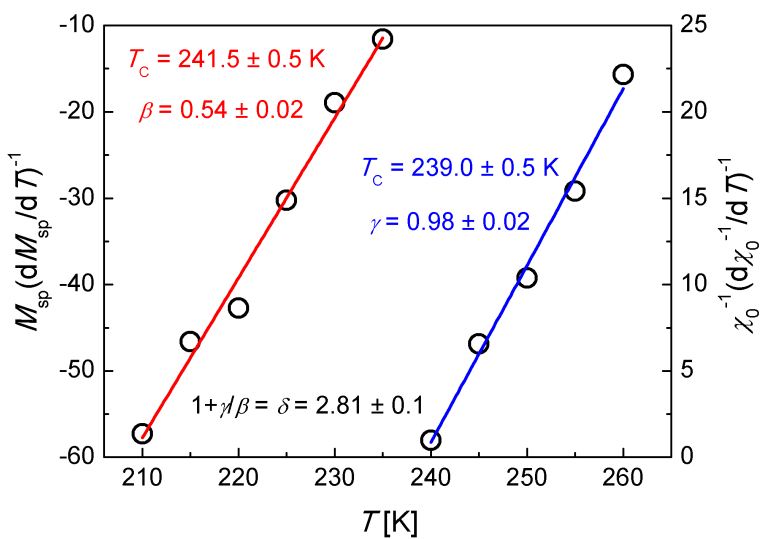

Fig. 3. Kouvel-Fisher plots (spontaneous magnetization $M_{s p}$ - left axis and the inverse initial magnetic susceptibility $\chi_{0}^{-1}$ - right axis) for $\mathrm{Y}_{8} \mathrm{Co}_{62} \mathrm{~B}_{30}$ amorphous alloy.

The Curie temperatures determined from $M_{s p}(T) /\left(\mathrm{d} M_{s p}(T) / \mathrm{d} T\right)$ and $\chi_{0}^{-1}(T) /\left(\mathrm{d} \chi_{0}^{-1}(T) / \mathrm{d} T\right)$ dependences are equal to $239.0 \pm 0.5 \mathrm{~K}$ and $241.5 \pm 0.5 \mathrm{~K}$, respectively and coincide very well with $T_{\mathrm{C}}$ values obtained from temperature dependences of $M_{s p}$ and $\chi_{0}^{-1}$ (Fig. 2). Critical exponent $\beta$, which describes the growth of ordered moment below $T_{\mathrm{C}}$, was determined to be equal to $0.54 \pm 0.02$, while $\gamma$, which expresses the divergence of the magnetic susceptibility at $T_{\mathrm{C}}$, is equal to $0.98 \pm 0.02$. Both exponents are close to mean-field model values $(\beta=0.5$ and $\gamma=1)$. Critical exponent $\delta=2.81 \pm 0.1$ determined using the Widom scaling relation (Eq. (4)) is also convergent with MF model $(\delta=3)$. This quantity is strictly connected with the curvature of $M(H)$ isotherm at the Curie temperature.

Divergence of the Curie temperature values determined with the use of $M(T)$ curves [2] with these obtained on the basis of the Arrott and Kouvel-Fisher plots can be associated with many different aspects. At most, a structural disorder can cause the shift and spread of $T_{\mathrm{C}}$ measured in different applied magnetic fields [12]. Moreover, application of modified Arrott plot [9], to obtain isotherms which are linear in broader $H$ range, would be helpful even assuming that the critical exponents indicate mean field model as the valid one.

Assuming determined Curie temperature for further considerations, the $M(H)$ isotherm at $240 \mathrm{~K}$ was selected for calculations of $\delta$ exponent on the basis of critical isotherm analysis (Eq. (3)). Critical isotherm mea- 
sured for $\mathrm{Y}_{8} \mathrm{Co}_{62} \mathrm{~B}_{30}$ amorphous alloy is plotted in Fig. 4 with $\ln M v s$. $\ln H$ plot in the inset. Slope of the linear fit of the high field region of the $\ln M(\ln H)$ plot gives $1 / \delta$. Small non-collinearity of isotherm causes deviation of $\delta$, which is equal to $2.60 \pm 0.01$, in comparison to the value determined on the basis of the Widom relation. Critical exponent would converge to MF value for the magnetic fields higher than considered fitting region ( $H>15 \mathrm{kOe})$. Nevertheless, the convergence is still high enough to count $\mathrm{Y}_{8} \mathrm{Co}_{62} \mathrm{~B}_{30}$ among MF model materials.

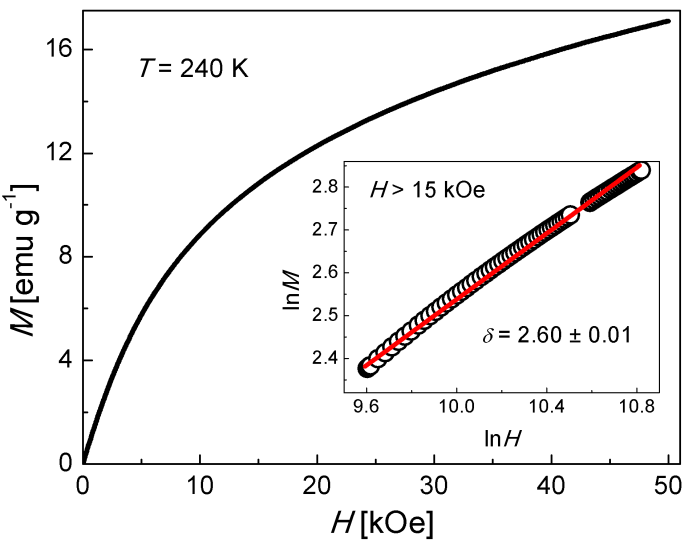

Fig. 4. Magnetization isotherm of $\mathrm{Y}_{8} \mathrm{Co}_{62} \mathrm{~B}_{30}$ amorphous alloy measured at critical temperature equal to $240 \mathrm{~K}$. Plot of $\ln M$ vs. $\ln H$ is shown in the inset with $\delta=2.60 \pm 0.01$ value obtained by the linear fit (solid red line) according to Eq. (3) for the high magnetic field region $(H>15 \mathrm{kOe})$.

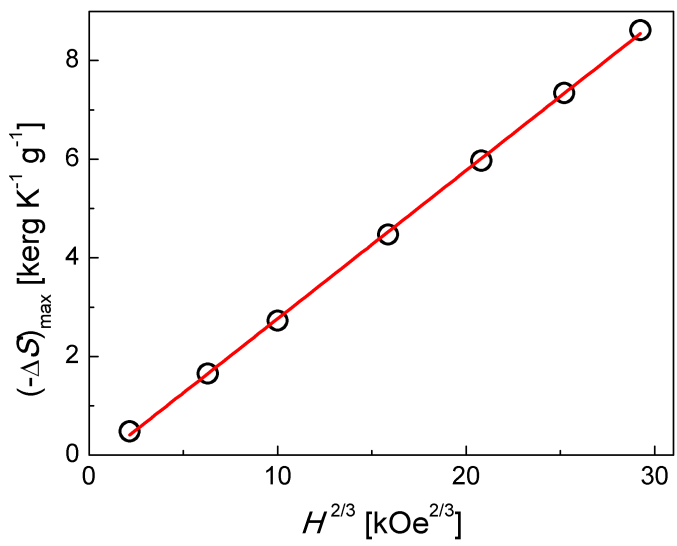

Fig. 5. Magnetic field dependence of maximum magnetic entropy change. Red line is the linear fit to experimental points.

The field dependence of the magnetic entropy change is shown in Fig. 5 and follows a power law with exponent equal to $2 / 3$, characteristic for mean-field approach in the range of moderate applied magnetic fields, as another evidence for validity of MF model in the investigated alloy [13]. Values of $(-\Delta S)_{\max }$ were determined on the basis of $\Delta S_{m}(T)$ curves shown before in Ref. [2].

All the results from utilized methods and the values of determined critical exponents are in good agreement with those of the MF model. It suggests that long-range ferromagnetic interactions are dominant in the alloy. Any observed discrepancies in obtained results are likely connected with non-linearity of isotherms. This is rather common that the scaling laws are not functioning properly below $T_{\mathrm{C}}$ and in low magnetic fields, especially. Magnetic inhomogeneities, which in case of amorphous structure are rather expected, play a significant role along with the influence of demagnetization factor. Its meaningful effect can be observed for instance in the Arrott plot, where the isotherms are decreasing asymptotically converging to non-zero $H / M$ values.

\section{Conclusions}

The magnetic transition observed in the $\mathrm{Y}_{8} \mathrm{Co}_{62} \mathrm{~B}_{30}$ alloy is of a second order type. Investigated system fulfils the criteria of mean field model. Accordingly, long range ferromagnetic interactions are representative feature of this amorphous alloy. These results were confirmed by the Arrott plot analysis, the Kouvel-Fisher method and the critical isotherm analysis, along with the Widom scaling relation and magnetic field dependence of maximum magnetic entropy changes.

\section{References}

[1] Y. Khan, R. Hawig, J. Therm. Anal. 38, 1585 (1992).

[2] Z. Śniadecki, J. Marcin, I. Škorvánek, N. Pierunek, B. Idzikowski, J. Alloys Comp. 584, 477 (2014).

[3] B. Idzikowski, A. Wrzeciono, J. Kováč, IEEE Trans. Magn. 30, 561 (1994).

[4] N. Pierunek, Z. Śniadecki, J. Marcin, I. Škorvánek, B. Idzikowski, IEEE Trans. Magn. 50, 2506603 (2014).

[5] P. Gębara, P. Pawlik, I. Škorvánek, J. Bednarcik, J. Marcin, S. Michalik, J. Donges, J.J. Wysłocki, B. Michalski, J. Magn. Magn. Mater. 372, 201 (2014).

[6] P. Nisha, S.S. Pillai, M.R. Varma, K.G. Suresh, J. Magn. Magn. Mater. 327, 189 (2013).

[7] X.C. Zhong, J.X. Min, Z.G. Zheng, Z.W. Liu, D.C. Zeng, J. Appl. Phys. 112, 033903 (2012).

[8] L. Zhang, J. Fang, J.Y. Fan, M. Ge, L.S. Ling, C.J. Zhang, L. Pi, S. Tan, Y.H. Zhang, J. Alloys Comp. 588, 294 (2014).

[9] R. Tlili, A. Omri, A. Dhahri, M. Bekri, M. Bejar, E. Dhahri, E.K. Hlil, J. Alloys Comp. 666, 425 (2016).

[10] B. Widom, J. Chem. Phys. 41, 1633 (1964).

[11] S.K. Banerjee, Phys. Lett. 12, 16 (1964).

[12] N.G. Bebenin, R.I. Zainullina, V.V. Ustinov, Y.M. Mukovskii, J. Magn. Magn. Mater. 324, 1112 (2012).

[13] V. Franco, A. Conde, Int. J. Refrig. 33, 465 (2010). 